\title{
Laser-driven formation of transient local ferromagnetism in FeRh thin films
}

DOI:

10.1016/j.ultramic.2017.03.024

\section{Document Version}

Accepted author manuscript

Link to publication record in Manchester Research Explorer

\section{Citation for published version (APA):}

Ünal, A. A., Parabas, A., Arora, A., Ehrler, J., Barton, C., Valencia, S., Bali, R., Thomson, T., Yildiz, F., \& Kronast, F. (2017). Laser-driven formation of transient local ferromagnetism in FeRh thin films. Ultramicroscopy. https://doi.org/10.1016/j.ultramic.2017.03.024

\section{Published in:}

Ultramicroscopy

\section{Citing this paper}

Please note that where the full-text provided on Manchester Research Explorer is the Author Accepted Manuscript or Proof version this may differ from the final Published version. If citing, it is advised that you check and use the publisher's definitive version.

\section{General rights}

Copyright and moral rights for the publications made accessible in the Research Explorer are retained by the authors and/or other copyright owners and it is a condition of accessing publications that users recognise and abide by the legal requirements associated with these rights.

\section{Takedown policy}

If you believe that this document breaches copyright please refer to the University of Manchester's Takedown Procedures [http://man.ac.uk/04Y6Bo] or contact uml.scholarlycommunications@manchester.ac.uk providing relevant details, so we can investigate your claim.

\section{OPEN ACCESS}




\title{
Laser-driven formation of transient local ferromagnetism in FeRh thin films
}

\author{
A. A. Ünal, ${ }^{1, a)}$ A. Parabas, ${ }^{2}$ A. Arora, ${ }^{1}$ J. Ehrler, ${ }^{3}$ C. Barton, ${ }^{4}$ S. Valencia, ${ }^{1}$ R. Bali, ${ }^{3}$ \\ T. Thomson, ${ }^{4}$ F. Yildiz, ${ }^{2}$ and F. Kronast ${ }^{1, a)}$ \\ ${ }^{1}$ Helmholtz-Zentrum Berlin für Materialien und Energie, Albert-Einstein-Str. 15, D-12489 Berlin, Germany \\ ${ }^{2}$ Gebze Teknik Üniversitesi, Temel Bilimler Fakültesi, Fizik Bölümü, TR-41400 Gebze/Kocaeli, Turkey \\ ${ }^{3}$ Institut für Ionenstrahlphysik und Materialforschung, Helmholtz-Zentrum Dresden-Rossendorf, D-01328 Dresden, Germany \\ ${ }^{4}$ School of Computer Science, University of Manchester, Manchester M13 9PL, United Kingdom
}

The antiferromagnetic to ferromagnetic phase transition in FeRh can be induced globally by either heating the material above its phase transition temperature or applying a combination of external stimuli (such as mechanical strain, electric/magnetic fields) on the material preheated close to its transition temperature. On the other hand, to locally induce this phase transition is more desirable for applications and requires a confined source of energy such as a focused laser beam. Here we combine laser excitation with X-ray magnetic imaging to determine the effect of laser heating on the local and transient magnetization of FeRh using time-resolved photoelectron emission microscopy. Excitation by an ultrashort laser pulse generates a local ferromagnetic state within $0.6 \mathrm{~ns}$ which recovers its initial antiferromagnetic state after a further $2 \mathrm{~ns}$. The form of the domains during the growth and diminution of ferromagnetic ordering suggests an intrinsic speed limit for magnetic and structural changes.

\section{INTRODUCTION}

The B2 chemically ordered phase of the $\mathrm{Fe}_{0.5} \mathrm{Rh}_{0.5}$ alloy undergoes a first order metamagnetic phase transition from antiferromagnetic (AF) to ferromagnetic (FM) as the temperature of the sample increases from room temperature to a critical temperature above $\sim 360 \mathrm{~K} .^{1,2}$ The transition is accompanied by an isotropic lattice expansion. At room temperature, the crystal structure of the equiatomic alloy is a $\mathrm{CsCl}$ type structure with one $\mathrm{Rh}$ atom at the center and eight nearest neighbor Fe atoms at the corners of a cubic unit cell; Fe spins order antiferromagnetically with $\pm 3 \mu_{B}$ and there is zero magnetic moment on the Rh atoms. ${ }^{3,4}$ Upon heating above the critical temperature, the FeRh lattice expands and the Fe atoms align ferromagnetically inducing a moment on $\mathrm{Rh}$ atoms.

The basic physical properties of bulk FeRh are well known. ${ }^{1,2}$ More recently there has been significant work on thin films with thicknesses of several 10's of nm. ${ }^{5,6}$ Incorporating FeRh into a more complex magnetic structure has attracted significant attention ${ }^{7,8}$ as a promising candidate for heat-assisted magnetic recording media: e.g. in an exchange-spring 
system with a hard magnetic layer such as FePt. ${ }^{9}$ In FeRh/FePt, the FM phase of FeRh (e.g. at $400 \mathrm{~K}$ ) lowers the switching coercive field of the magnetically hard FePt layer by a spring exchange mechanism. However, when the temperature is lowered to room temperature, the AF phase of FeRh does not alter the coercive field of the hard layer, which stabilizes the magnetic state and hence the recorded data. The low phase transition temperature of FeRh allows the exchange spring mechanism to be realized within a temperature window of just $\Delta T=100 \mathrm{~K}$. This is significantly lower than the temperature difference of $\Delta T=450 \mathrm{~K}$, which is the difference between room temperature and the Curie temperature of FePt $\left(T_{C}=750 \mathrm{~K}^{10}\right)$. Similarly, FeRh was proposed in different bilayer systems that utilize its phase transition temperature, i.e. the Neél temperature, for inducing the exchange bias effect. ${ }^{11,12}$ FeRh thin films have also recently attracted new technological interest due to the expansion of its crystal lattice that accompanies the magnetic phase transition. The unit cell volume increases by $\sim 1 \%$ from the AF to the FM state, ${ }^{13}$ which makes the FeRh system a promising material when coupled to ferroelastic and ferroelectric materials. ${ }^{14,15}$ This potential was recently demonstrated by experiments on the $\mathrm{FeRh} / \mathrm{BaTiO}_{3}$ system where it was shown that the magnetic order of a FeRh thin film can be controlled electrically via the voltage-induced strain from the $\mathrm{BaTiO}_{3}$ substrate ${ }^{16}$.

To investigate the dynamics of the structural and magnetic changes in the FeRh lattice, there have been a number of time-resolved studies on FeRh using experimental techniques like transient reflectivity, X-ray diffraction, magnetooptical Kerr effect and X-ray magnetic circular dichroism experiments. ${ }^{17,18,19,20,21,22}$ In summary, various contradictory time scales from sub-ps ${ }^{17,18}$ up to $30 \mathrm{ps}^{19,21}$ were reported for rapid magnetization processes, and slower time scales from several hundred picoseconds $\mathrm{s}^{20,21}$ to a nanosecond ${ }^{22}$ were reported for the magnetic phase transition and the concomitant crystal lattice expansion to occur. Despite numerous investigations of the laser-induced magnetic/structural phase transition dynamics, magnetic domain imaging of the laser focus region has never been observed directly; in fact this is a challenging task that requires a setup with time-resolved imaging combining tight laser focusing. Due to the lack of spatial information in the experiments above, there have been many speculative scenarios regarding how the magnetic domains should appear and disappear, and there is a vague state of understanding of the phase transition dynamics. Combination or comparison of time scales from the experiments mentioned above is also difficult as all experiments have different sample thicknesses, some with heat sinks, capping layers etc.

Here we unite both spatial and temporal information in one experiment and present the first laser-induced magnetization experiments on FeRh. We present the generation and disappearance of FM order in thin films of FeRh via time-resolved optical pump and X-ray probe experiments in a photoelectron emission microscope. Focusing a laser beam to a spot size of a few microns heats the FeRh lattice and induce the AF-to-FM phase transition. We show the extent of single pulse laser heating and its effect on the magnetization of the sample in both the spatial and temporal domains $\boldsymbol{M}(\boldsymbol{r}, t)$. Our results indicate the time scales of magnetization growth and lattice expansion in terms of magnetic domain sizes/shapes during laser heating and cooling. We believe our results to be also very interesting for future 
technological applications, because the presented time-resolved magnetic imaging proves a direct read/write control over tiny magnetic entities.

\section{EXPERIMENTAL}

Equiatomic FeRh(001) thin films of $33 \mathrm{~nm}$ thickness were grown by dc magnetron sputtering from an FeRh target on a $\mathrm{MgO}(001)$ substrate. The magnetic phase transition of the film was macroscopically characterized by a vibrating sample magnetometer (VSM) and locally using X-ray magnetic circular dichroism (XMCD) in combination with an Xray photoelectron emission microscope (X-PEEM). Figure 1(a) shows the $M(T)$ magnetization hysteresis as a function of sample temperature using a $5 \mathrm{~T}$ external magnetic field. Heating and cooling cycles clearly show the magnetic phase transition of the FeRh film (from the VSM measurement). The heating curve shows that the sample becomes FM above $360 \mathrm{~K}$. The FeRh unit cells with AF and FM magnetic ordering are represented in the insets of Fig. 1(a).

High-resolution images of the magnetic domain configurations (50 $\mathrm{nm}$ spatial resolution) across the phase transition were obtained by XPEEM ${ }^{23}$ at the UE49-PGM1a beamline of the BESSY II synchrotrom. Temperaturedependent XMCD images were obtained by tuning the synchrotron photon energy to the $L_{3}$ resonance edge of iron (707.5 eV), thus exploiting the element selectivity of X-rays. Each of the XMCD images was calculated from a sequence of X-ray absorption images taken with alternating left and right helicity circular polarization. The difference between two images with opposite helicity were divided by their sum to yield the so-called XMCD asymmetry which shows a projection of the iron magnetic domain contrast on the incidence direction of the x-ray beam.

For the laser experiments, we used a normal incidence laser excitation geometry that results in a diffraction limited spot size of around $3 \mu \mathrm{m}$ by $5 \mu \mathrm{m}^{24}$ [Fig. 1(b) inset]. In this setup a sample holder with integrated optical components focuses the pump laser beam (770-830 nm wavelength, $100 \mathrm{fs}$ pulse duration, $5 \mathrm{MHz}$ repetition rate) into the sample from the backside, i.e. through the double side polished, transparent $\mathrm{MgO}$ substrate. Magnetic changes induced by the laser pulse heating on the sample are probed both in time and space using the XMCD technique described above. Figures $1(\mathrm{c}-\mathrm{d})$ present a simulation of heat distribution (using the heat conduction equation) in FeRh corresponding to the laser excitation geometry of Fig. 1(b) using a laser pulse with a fluence of $1.7 \mathrm{~mJ} / \mathrm{cm}^{2}$. For the XPEEM relevant time scales - on the order of hundreds of picoseconds - it is not necessary to have femtosecond temporal resolution in the simulation; therefore, we integrate over a time step of $20 \mathrm{ps}$ and do not account for ultrafast electron-phonon couplings. Additionally, because the laser light excites the sample from its backside, surface properties of the sample like capping or oxidation layers are not relevant for our simulation. All material properties such as heat capacity, thermal diffusivity, etc. are used from the literature resources. The simulation suggests that tight focused laser pulses of certain energy density can heat the laser spot region above the FeRh magnetic phase transition temperature for a temporal window more than a nanosecond, which makes a time-resolved XMCD experiment feasible. 
For the time-resolved experiments the repetition rate of the laser is set to $1.25 \mathrm{MHz}$ to match the X-ray pulse frequency in the so-called multi bunch operation mode of BESSY II with a single camshaft bunch in the center of the clearing gap of the multi bunch filling. ${ }^{25}$ To ensure a true time-resolved signal, the electron detector of the XPEEM is gated so that only those photoelectrons emitted by the X-ray camshaft bunch are counted. Temporal resolution achieved by this setup is limited to $50 \mathrm{ps}$ due to the X-ray pulse width of 30-50 ps. Time-resolved magnetic imaging requires a stroboscopic measurement and therefore requires a reproducible FM domain configuration each time the laser pulse heats the sample above its phase transition temperature. For this purpose, we apply a small external magnetic bias field (approx. $5 \mathrm{mT}$ ) to ensure a stable single domain configuration. For a fixed laser pump - X-ray probe delay time $(\Delta \mathrm{t})$, the laser pulse heats the sample spot above its phase transition temperature (see Fig. 1(d)) which results in the formation of a single-domain state along the external magnetic field direction. The area heated by the laser then cools and the AF ordering is recovered as thermal equilibrium is reached and the system is ready for another cycle of laser-driven AFM$>$ FM- $>$ AFM ordering. The process is then continually repeated to collect statistically meaningful data.

\section{RESULTS}

Figures 2(a-d) show static XMCD-PEEM images for four different sample temperatures. Almost zero XMCD contrast at room temperature indicates AF ordering. Upon heating the sample up to $380 \mathrm{~K}$, FM domains start nucleating in the AF matrix [Fig. 2(b)] and become more pronounced at $390 \mathrm{~K}$ [Fig. 2(c)]. Blue and red regions indicate positive and negative XMCD contrast and arise from FM domains with a magnetization component parallel or antiparallel to the incoming X-rays, respectively. White regions with zero XMCD contrast represent mainly the AF phase, or a FM phase with magnetization perpendicular to the incoming X-rays. At 410K, Fig. 2(d), the sample becomes fully FM, and after applying and removing the external magnetic field of $5 \mathrm{mT}$, the sample turns into a single domain state as seen in Fig. 2(e) with a maximum XMCD asymmetry of around 6\%. Cooling the sample back to the room temperature restores the AF phase. ${ }^{26}$

Time-resolved XMCD-PEEM images are presented in Figs. 3(a-1), starting from negative time delays up to a delay of $2.07 \mathrm{~ns}$ as labeled on each image. At negative time delays, i.e., X-ray probes the sample before it is heated by the laser pulse there is no XMCD contrast, as expected. At time-zero $(\Delta t=0 \mathrm{~ns})$, we start observing a faint but homogenous XMCD contrast on a region larger than the laser excited area pointing to the formation of FM domains. The local extent of these domains is larger than the laser beam profile seen in the inset of Fig. 3(1). Contrary to the temperature simulations of Fig. 1(c-d), the induced XMCD is homogenous and does not replicate the Gaussian beam profile of the laser beam. XMCD contrast increases as a function of delay time and maximum XMCD signal (as high as $6 \%$ as shown in Fig. 2(e)) is established for $\Delta t=0.69$ ns. The signal decays at longer time delays concomitant with a shrinking of the FM domain size until it disappears at $\Delta \mathrm{t}=2.07 \mathrm{~ns}$. For a more detailed analysis we extracted from each magnetic image 
a line profile along the grey line indicated in Fig. 3(a). These line profiles are plotted as a function of delay in Fig. 3(mn) and demonstrate a quantitative comparison for the growth and disappearance of the locally induced magnetization.

A line profile across the laser spot on the sample (thin black line in Figs. 3(m-n), extracted from the laser spot image from the inset of Fig. 3(1)) serves as a reference for the local laser beam intensity. The first line profile from the $\mathrm{XMCD}$ signal of $\Delta \mathrm{t}=0 \mathrm{~ns}$ shows a plateau-like intensity distribution within the laser spot, indicating a uniform XMCD contrast in the developing magnetic domain. The plateau height increases at longer delay times until the XMCD line profile resembles that of a Gaussian distribution similar to the laser spot line profile when the delay time exceeds $\Delta \mathrm{t}=$ $0.69 \mathrm{~ns}$. Time delays from $\Delta \mathrm{t}=0.97 \mathrm{~ns}$ to $2.07 \mathrm{~ns}$ show a gradual decrease in ferromagnetic ordering, with the XMCD line profiles continuing to resemble that of the laser spot.

Comparing the evolution of the XMCD line profile to the local Gaussian laser beam intensity and the simulated evolution of the laser induced temperature profile in Fig. 1, we find a clear discrepancy within the first 0.69 ns time window. XMCD signal starts appearing with a plateau-like intensity onset and builds up steadily as a function of the time delay. This finding highlights an intrinsic time delay for the buildup time of magnetic phase transition in the FeRh system. If the magnetic phase transition were to happen instantaneously with temperature, we would expect the maximum XMCD contrast to occur right after thermalization in the laser spot center at time delays below $0.1 \mathrm{~ns}$ However, the data show an increasing XMCD signal which evolves initially at a constant level within the laser spot, regardless of local variations in the Gaussian laser fluence. It takes 0.69 ns to reach the maximum XMCD signal in the center of the laser spot and it is only after this time delay that the local magnetization matches the laser induced temperature profile. These findings provide direct evidence that the magnetic phase transition in FeRh occurs over a finite time and the involving time-constant does not vary with the local fluence.

Plotting XMCD signals as a function of time delay from different lateral positions (not shown here) show that the region 2-3 $\mu \mathrm{m}$ away from the laser spot center reaches an XMCD maximum between $\Delta \mathrm{t}=0.42-0.69 \mathrm{~ns}$ (see curves in Fig. 3(m) for the lateral positions of $4 \mu \mathrm{m}$ and $9 \mu \mathrm{m}$, for instance). A similar observation can be made when we integrate all region to converge the case of spatially averaging experiments: the XMCD maximum is achieved after approximately $\Delta \mathrm{t}=0.42 \mathrm{~ns}$, the signal decreases slowly up to $0.69 \mathrm{~ns}$; overall the growth resembles an S-shaped curve with an exponential time constant of $0.5 \mathrm{~ns}$, then a more pronounced exponential decay starts. We attribute this growth time constant to the speed of the FeRh magnetic phase transition and the concomitant crystal lattice expansion. This time delay could be due to the intrinsic time taken for the crystal lattice to expand, which is expected to evolve on the order of the speed of sound, i.e. $5 \mu \mathrm{m} / \mathrm{ns},{ }^{21}$ corresponding to our spatial and temporal dimensions. The time required to reach the ferromagnetic state is consistent with some of the previous spatially averaging experiments, ${ }^{20,21}$ but considering the differences in sample thicknesses and the used heat sinks and capping layers etc. it is not possible to 
extract the sole FeRh dynamics from the rest of parameters that affect the time scales. Due to our temporal resolution of

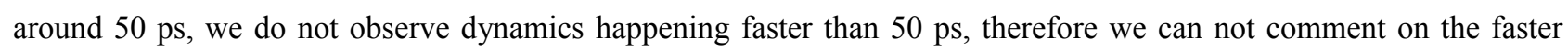
dynamics.

In conclusion, we presented the first experimental results combining temporal and spatial dimensions that demonstrate the existence of an intrinsic time for the magnetic phase transition in FeRh, even on very small length scales. Thanks to this unique technique, we find that there is an intrinsic speed limit for the FM state to be established at the $100 \mathrm{~nm}$ scale within time scale on the order of $0.5 \mathrm{~ns}$. The transient magnetic state determined here shows that it is possible to modify the magnetization of the sample in a very short time interval $(<0.7 \mathrm{~ns})$ and that it is only limited by the intrinsic speed of the FeRh magnetic phase transition. The time frame of the phase transition is compatible with magnetic recording technologies where a new magnetic area/bit can be written every 1-2 ns. Assuming that in the near future, plasmonics and near-field optics will focus and transmit optical energy to spot sizes of around $(25-50 \mathrm{~nm})^{2}$, memory devices based on exchange spring systems with FeRh could be used as the next generation magnetic recording media. $^{27}$

\section{ACKNOWLEDGMENTS}

We acknowledge the support of Helmholtz-Zentrum Berlin, and L. Gierster for his work on the optical sample holder. The support of the UK EPSRC through grant number EP/K008412/1 is acknowledged. 
FIG. 1

FIG. 1. (a) Magnetization versus temperature curve of the FeRh film across the antiferromagnetic to ferromagnetic phase transition. (b) Laser excitation geometry in the XPEEM. Focused laser beam excites the thin film sample from its backside and X-ray beam probes the change in magnetization from the top side. Inset shows a PEEM image of photoelectrons emitted from the sample surface due to the focused laser pulse excitation. (c) Simulation showing heat distribution in the sample as a function of lateral distance and time after absorption of a focused laser pulse at time-0. (d) Induced temperature curves for selected time delays.
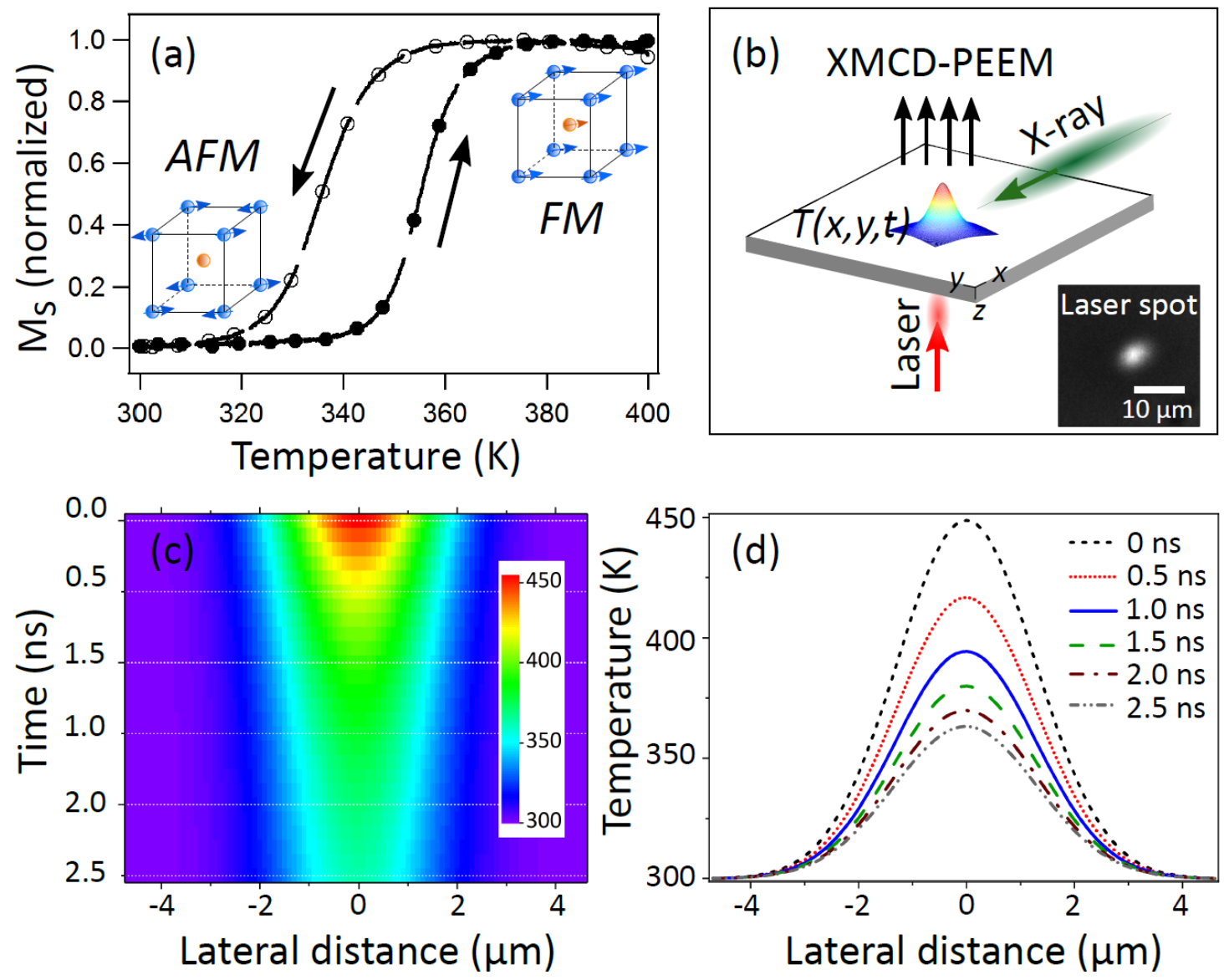
FIG. 2

FIG. 2. (a-d) Fe XMCD-PEEM images taken as a function of temperature during heating, the antiferromagnetic state at room temperature turns into ferromagnetic state as the sample is heated. (e) An external magnetic field pulse turns the multi domain state into a single domain state with a uniform $6 \% \mathrm{XMCD}$ asymmetry. Experimental geometry and the incoming x-ray beam relative to the orientation of the XMCD-PEEM images are also shown.

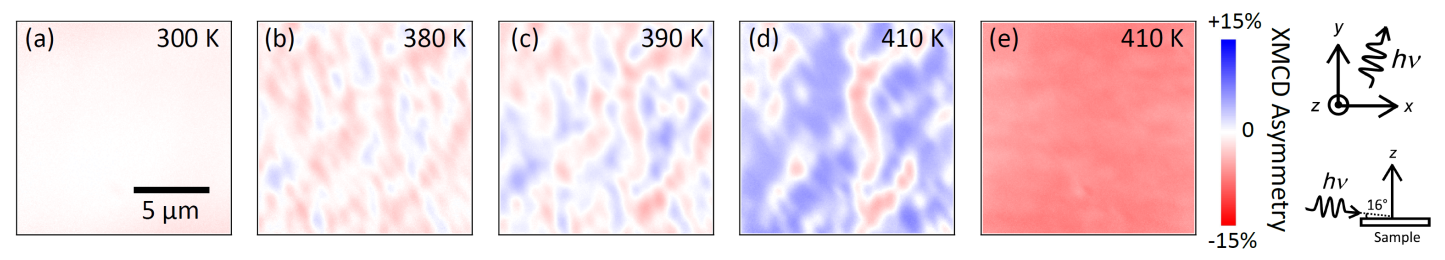


FIG. 3

FIG. 3. (a-1) Magnetic images of the sample at the Fe $L_{3}$ edge for different pump-probe time delays. Inset of Fig. 3(1): laser spot image on the sample for comparison. Scale bar for all images is $5 \mu \mathrm{m}$. (m-n) Line profiles from several time delays stacked up together for shape and intensity comparison: (m) during laser heating, (n) during cooling. Thin black line is the profile of the laser spot on the sample extracted using the same line profile from Fig. 3(a) to compare with the magnetic profiles.
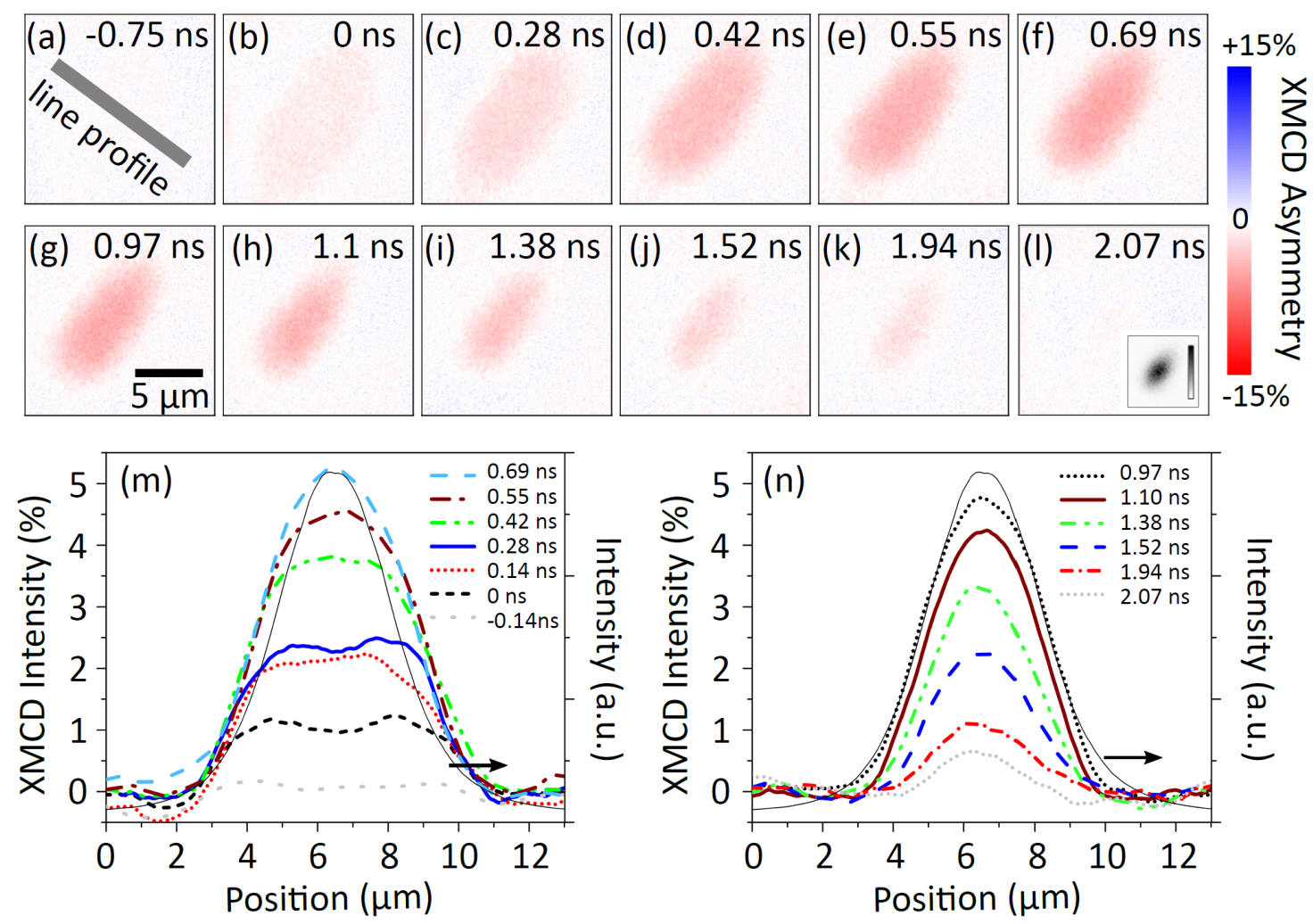
A. Akin Ünal: uenal@mbi-berlin.de

Present address: Max Born-Institute for Nonlinear Optics and Short Pulse Spectroscopy, Max-Born-Str. 2A, 12489 Berlin, Germany

F. Kronast: florian.kronast@helmholtz-berlin.de

M. Fallot and R. Hocart, Rev. Sci. 77, 498 (1939).

J. S. Kouvel and C. C. Hartelius, J. Appl. Phys. 33, 1343 (1962).

G. Shirane, R. Nathans, and C. W. Chen, Phys. Rev. 134, A1547 (1964).

L. M. Sandratskii and P. Mavropoulos, Phys. Rev. B 83, 174408 (2011).

C. Stamm, J.-U. Thiele, T. Kachel, I. Radu, P. Ramm, M. Kosuth, J. Minár, H. Ebert, H. A. Dürr, W. Eberhardt, and C. H. Back, Phys. Rev. B 77, 184401 (2008).

C. Baldasseroni, C. Bordel, A. X. Gray, A. M. Kaiser, F. Kronast,J. Herrero-Albillos, C. M. Schneider, C. S.

Fadley, and F. Hellman, Appl. Phys. Lett. 100, 262401 (2012).

X. Marti et al., Nat. Mater. 13, 367 (2014).

J. U. Thiele, S. Maat, and E. E. Fullerton, Appl. Phys. Lett. 82, 2859 (2003).

F. Garcia-Sanchez, O. Chubykalo-Fesenko, O. Mryasov, R. W. Chantrell, and K. Yu. Guslienko, Appl. Phys. Lett. 87, 122501 (2005).

M. H. Kryder, E. C. Gage, T. W. McDaniel, W. A. Challener, R. E. Rottmayer, G. Ju, Y.-T. Hsia, and M. F. Erden, Proc. IEEE 96, 1810 (2008).

N. T. Nam, W. Lu, and T. Suzuki, J. Appl. Phys. 105, 07D708 (2009).

I. Suzuki, Y. Hamasaki, M. Itoh, and T. Taniyama, Appl. Phys. Lett. 105, 172401 (2014).

A. I. Zakharov, A. M. Kadomtseva, R. Z. Levitin, and E. G. Ponyatovskii, Soviet Phys. JETP 19, 1348 (1964). Y. Lee et al., Nat. Comm. 6, 5959 (2015).

R. O. Cherifi et. al., Nat. Mater. 13, 345 (2014).

L. C. Phillips et al., Scientific Reports 5, 10026 (2015).

G. Ju, J. Hohlfeld, B. Bergman, R. J. M. van de Veerdonk, O. N. Mryasov, J.-Y. Kim, X. Wu, D. Weller, and B. Koopmans, Phys. Rev. Lett. 93, 197403 (2004).

J. U. Thiele, M. Buess, and C. H. Back, Appl. Phys. Lett. 85, 2857 (2004).

B. Bergmann, G. Ju, J. Hohlfeld, R. J. M. van de Veerdonk, J.-Y. Kim, X. Wu, D. Weller, and B. Koopmans, Phys. Rev. B 73, 60407(R) (2006).

I. Radu, C. Stamm, N. Pontius, T. Kachel, P. Ramm, J. U. Thiele, H. A. Dürr, and C. H. Back, Phys. Rev. B 81, $104415(2010)$.

21 S. O. Mariager, F. Pressacco, G. Ingold, A. Caviezel, E. Möhr-Vorobeva, P. Beaud, S. L. Johnson, C. J. Milne, E. Mancini, S. Moyerman, E. E. Fullerton, R. Feidenhans'l, C. H. Back, and C. Quitmann, Phys. Rev. Lett. 
108,87201 (2012).

22 F. Quirin, M. Vattilana, U. Shymanovich, A. El-Kamhawy, A. Tarasevitch, J. Hohlfeld, D. von der Linde, and K. Sokolowski-Tinten, Phys. Rev. B 85, 20103(R) (2012).

23 F. Kronast, J. Schlichting, F. Radu, S. K. Mishra, T. Noll, and H. A. Dürr, Surf. Interface Anal. 42, 1532 (2010).

24 L. Gierster, L. Pape, A. A. Ünal, and F. Kronast, Rev. Sci. Instrum. 86, 23702 (2015).

25 http://www.helmholtz-berlin.de/quellen/bessy/betrieb-beschleuniger/betriebsmodi_en.html

26 S. O. Mariager, L. Le Guyader, M. Buzzi, G. Ingold, and C. Quitmann, arXiv:1301.4164 (2013).

27 T. W. McDaniel, J. Appl. Phys. 112, 093920 (2012). 